\title{
Allesfresser im Kinosaal? Distinktion durch kulturelle Vielfalt in Deutschland $^{1}$
}

\author{
Von Jörg Rössel
}

Zusammenfassung: Zur Beschreibung des kulturellen Wandels in postindustriellen Gesellschaften wird in zunehmendem Maße auf die in den Vereinigten Staaten vor allem von Richard A. Peterson formulierte These von der Entwicklung des kulturellen Allesfressers (Cultural Omnivore) zurückgegriffen. Deren Kerngehalt ist die Annahme, dass eine ausschließliche Orientierung an der klassischen Hochkultur in gegenwärtigen Gesellschaften ihre soziale Funktion - z. B. im Bildungssystem und auf dem Arbeitsmarkt - verloren hat und dass an diese Stelle eine Vorliebe für die kulturelle Vielfalt getreten ist, die neben der Hochkultur auch Elemente der populären Kultur einschließt. In diesem Aufsatz werden erstens drei verschiedene Interpretationen dieses Phänomens vorgestellt, zweitens die bisherige empirische Evidenz zur Übertragbarkeit des Konzepts auf Deutschland kritisch diskutiert und drittens eine eigene empirische Analyse auf der Basis einer Umfrage unter Kinobesuchern dargestellt. Obwohl die Sparte des Films eigentlich dem Phänomen der kulturellen Allesfresserei besonders entgegenkommt, ergibt sich nur eine schwache Evidenz für die Verwendbarkeit des Konzepts in Deutschland.

\section{Einleitung}

Zur Beschreibung des kulturellen Wandels in postindustriellen Gesellschaften wird in zunehmendem Maße auf die in den Vereinigten Staaten vor allem von Richard A. Peterson formulierte These von der Entwicklung des kulturellen Allesfressers (Cultural Omnivore) zurückgegriffen (Peterson 1992; 1997; Peterson/Simkus 1992; Peterson/Kern 1996). Der Kerngehalt dieser These ist die Annahme, dass eine ausschließliche Orientierung an der klassischen Hochkultur in gegenwärtigen Gesellschaften ihre soziale Funktion - z. B. im Bildungssystem und auf dem Arbeitsmarkt - verloren hat (vgl. Gebesmair 2004) und dass an diese Stelle eine Vorliebe für die kulturelle Vielfalt getreten ist, die neben der Hochkultur auch Elemente der populären Kultur einschließt. Auch im deutschsprachigen Raum wurde die These vielfach rezipiert (Gebesmair 1998; 2001; Hartmann 1999; Neuhoff 2001). Während sie von Gebesmair als innovative Konzeption zur Erfassung des kulturellen Wandels betrachtet wird, hat Neuhoff in einer Studie die Übertragbarkeit dieses Konzepts auf die deutsche Gesellschaft empirisch analysiert. Seine Resultate stellen die Verwendung der Allesfresser-These für die Beschreibung von Prozessen des kulturellen Wandels in Deutschland sehr deutlich in Frage. Insofern soll genau diese Diskussion im vorliegenden Aufsatz aufgenommen werden. In einem ersten Schritt werden drei Lesarten der Allesfresser-These kurz skizziert: erstens die Analysen von Peterson selbst, die empirisch stark auf die Vielzahl von präferierten populären Genres fokussieren, zweitens die Arbeiten des australischen Sozialwissenschaftlers Michael Emmison (2003), der mit seinem Begriff der kulturellen Mobilität weniger auf die Präferenz für eine Vielzahl von Genres und kulturellen Richtungen abzielt, sondern vor allem auf die Kenntnis einer Vielfalt von kulturellen Phänomen, und schließlich drittens Bethany Brysons Studien, die auch weniger die kulturellen Präferenzen, sondern vor allem die kulturelle Toleranz im Blick haben (1996). Im Anschluss an diese Exposition der theoretischen Konzepte soll die empirische Aussagefähigkeit der Studie von

1) Für fruchtbare weiterführende Hinweise sei an dieser Stelle Andreas Gebesmair, Gunnar Otte und dem anonymen Gutachter der Sozialen Welt gedankt. Für die ausgezeichnete Kooperation bei der Durchführung der Befragung möchte ich Kathi Bromberger meinen Dank aussprechen. Finanziert wurde die Studie von der Jungen Akademie.

Soziale Welt 57 (2006), S. $259-272$ 
Neuhoff, der vor allem die von Peterson und Bryson entwickelten Lesarten des Allesfresserphänomens betrachtet hat, noch einmal genauer unter die Lupe genommen werden. Dabei wird gezeigt werden, dass seine Stichprobe so stark verzerrt war, dass damit keine Aussage über die Anwendbarkeit des Allesfresserphänomens in Deutschland möglich war. Dies wird auch in einer Sekundäranalyse des ALLBUS von 1998 deutlich, deren Resultate von Neuhoffs Ergebnissen in erheblichem Maße abweichen.

Im empirischen Teil des Aufsatzes wird eine Besucherumfrage im Kinopublikum vorgestellt, die eine empirische Operationalisierung aller drei Lesarten der These vom kulturellen Allesfresser ermöglicht. Zudem wird damit die Allesfresser-These für einen kulturellen Bereich analysiert, der diesem Konzept in besonderem Maße entgegenkommt, da Filmgenres weniger stark hierarchisiert sind als Musikarten und Kinobesucher darüber hinaus einen relativ niedrigen Altersdurchschnitt haben. Sollten sich selbst in einer Studie von Kinobesuchern keine Hinweise für die Anwendbarkeit der Allesfresser-These in Deutschland finden, so kann man wohl die Suche nach weiterer empirischer Evidenz aufgeben.

\section{Drei Lesarten der These vom kulturellen Allesfresser und ihre empirische Evidenz}

\subsection{Die These von Peterson und Kern}

Die ersten Hinweise im Hinblick auf die Allesfresser-These ergaben sich in einer Studie von Peterson und Simkus (1992), in der die beiden Autoren den Zusammenhang zwischen Berufsgruppenzugehörigkeit und Musikvorlieben in den Vereinigten Staaten analysiert haben. Dabei konnten sie feststellen, dass die Berufsgruppen am oberen Ende der Statusdimension sich keineswegs nur für hochkulturelle Musikrichtungen wie klassische Musik oder Opernmusik interessierten, sondern auch für eine Vielzahl von populären Musikrichtungen. Dagegen zeigte sich am unteren Ende der Berufsskala, dass die Befragten sich sehr viel stärker mit einer einzigen - meist populären - Musikrichtung identifizieren konnten (Peterson/Simkus 1992). Mit den klassischen sozialwissenschaftlichen Vorstellungen von sozialer Distinktion durch die Orientierung an Hochkultur oder legitimer Kultur, wie man sie z.B. bei Pierre Bourdieu (1982) findet, ließen sich diese Ergebnisse kaum vereinbaren. Diese empirischen Evidenzen haben daher Peterson und Kern in einem 1996 erschienenen Artikel zur bekannten Allesfresser-These weiterentwickelt. Der Kern dieser These ist, dass hochkulturorientierte Amerikaner in immer geringerem Maße ausschließlich an Hochkultur interessiert, also sogenannte Hochkultursnobs sind, sondern immer stärker daneben auch ein Interesse für populäre kulturelle Genres entwickelt haben (Peterson/Kern 1996). In einem Vergleich von Umfragedaten aus den Jahren 1982 und 1992 können die beiden Autoren wiederum am Beispiel des Musikgeschmacks zeigen, dass die Befragten generell zum späteren Zeitpunkt eine Vorliebe für eine größere Vielfalt von musikalischen Genres zeigten. Dies gilt aber in besonderem Maße für die hochkulturorientierten Personen. ${ }^{2}$ Im Jahre 1982 mochten diese von 8 populären Musikrichtungen im Durchschnitt 3,72, im Jahr 1992 aber schon 4,35. Bei den anderen Personen lässt sich nur ein Anstieg von 2,81 auf 3,19 feststellen (Peterson/Kern 1996, S. 902). Insofern zeigt sich empirisch ein genereller Trend zur kulturellen Vielfalt, doch dieser ist bei den hochkulturorientierten Personen stärker vorhanden.

Peterson und Kern lokalisieren diesen kulturellen Wandel in den sozialen Veränderungen der amerikanischen Gesellschaft in den vergangenen Jahrzehnten. Dabei verweisen sie auf strukturelle Veränderungen, wie den Wohlstandszuwachs, die Bildungsexpansion, erhöhte

2) Dies wurde operationalisiert durch eine Vorliebe für Klassik und Oper, wobei zusätzlich eine der beiden Musikrichtungen als bevorzugte Richtung ausgewählt werden musste (Peterson/Kern 1996, S. 900). 
geographische und soziale Mobilität, die die soziale Grundlage für hochkulturelle Exklusion haben erodieren lassen (Peterson/Kern 1996, S. 905). Auch in der kulturellen und künstlerischen Sphäre sind die Grundlagen für elitären Snobismus weggebrochen: einerseits führt der postmaterialistische Wertewandel zu einer größeren Toleranz gegenüber kultureller Vielfalt, andererseits hat sich die avantgardistische Kunst in eine Richtung entwickelt, die die Formulierung von klaren ästhetischen Maßstäben für den Ausschluss von populärer Kultur immer weniger erlaubt. Schließlich sind auch die jüngeren Generationen durch eine Populärkultur sozialisiert, die sich als echte Alternative zur traditionellen Hochkultur verstanden hat und die damit auch den Status der klassischen Kultur angreift (Peterson/Kern 1996, S. 905-906). Zugleich sehen Peterson und Kern aber auch eine klassenstrukturelle Grundlage für den Wandel vom Hochkultursnobismus hin zur kulturellen Allesfresserei: »As highbrow snobbishness fits the needs of the earlier entrepreneurial upper-middle class, there also seems to be an elective affinity between today's new business-administrative class and omnivorousness « (Peterson/Kern 1996, S. 906). Gerade aus dieser Verbindung von kulturellen Wandlungsprozessen zu sozialstrukturellen Veränderungen bezieht die These von der kulturellen Allesfresserei wohl ihren Reiz. Denn sie erlaubt auch für moderne, differenzierte Gesellschaften die Analyse von Prozessen sozialer Distinktion, die aber eben nicht mehr ausschließlich an Hochkultur festzumachen ist, sondern an einer Vorliebe für kulturelle Vielfalt, die freilich auch immer die hochkulturellen Genres einschließt.

\subsection{Michael Emmisons Konzept der kulturellen Mobilität}

In den empirischen Analysen von Peterson und Kern lag der Fokus immer auf kultureller Vielfalt im Sinne der Anzahl von musikalischen Genres, die eine Person schätzt (Peterson/ Kern 1996, S. 901). Sie selbst weisen aber schon darauf hin, dass kulturelle Allesfresserei nicht notwendigerweise bedeuten muss, dass hochkulturorientierte Personen nun plötzlich alle musikalischen Genres gleichermaßen mögen, sondern dass diese Personen vor allem offen für Vieles sind (Peterson/Kern 1996, S. 904). So plausibel dieser Hinweis ist, so erfordert er doch eine etwas andere theoretische und empirische Vorgehensweise, die man vor allem in Arbeiten von Michael Emmison (2003) findet. Dieser verweist darauf, dass der Begriff des kulturellen Allesfressers in zu starkem Maße eine wahllose Vorliebe für eine breite Vielfalt an kulturellen Genres suggeriert und damit einen wichtigen Aspekt des kulturellen Lebens in modernen Gesellschaften außer Acht lässt: Emmison betont nämlich, dass kulturelle Fähigkeiten und Kenntnisse in unterschiedlichen Kontexten strategisch eingesetzt werden können. So muss ein technischer Angestellter im Gespräch mit seinen Kollegen andere kulturelle Kenntnisse und Kompetenzen mobilisieren als in der Diskussion mit seinen Nachbarn oder den kunstbeflissenen Akademikerfreunden seiner Frau (vgl. dazu auch DiMaggio 1987). Insofern kommt es aus Emmisons Perspektive für die strategische Nutzung von kulturellen Kompetenzen in verschiedenen sozialen Netzwerken nicht in erster Linie darauf an, dass eine Person eine tatsächliche Vorliebe für zahlreiche Genres gleichermaßen hat, sondern vor allem darauf, dass eine Person über zahlreiche künstlerische Richtungen informiert ist und sich darüber unterhalten kann. Daher verwendet er auch den Begriff der kulturellen Mobilität.

Auf der Basis von in Australien erhobenen Daten (vgl. dazu auch Bennett/Emmison/Frow 1999) untersucht Emmison vergleichend das Allesfresserkonzept von Peterson und Kern und sein eigenes Konzept der kulturellen Mobilität. Anders als Peterson und Kern behaupten, findet sich unter den Angehörigen der hochqualifizierten Klassenlagen nur eine sehr begrenzte Anzahl von kulturellen Allesfressern, dagegen kann er zeigen, dass diese Gruppen sich sowohl in hochkulturellen musikalischen Genres auskennen wie auch in populären musikalischen Genres. Arbeiter und einfache Angestellte verfügen dagegen zwar über eine breite Kenntnis populärer Musik, sind aber von den klassischen Genres nahezu ausgeschlossen. 
»Manual workers remain more ore less confined to a >lowbrow< musical world in both their tastes and expertise; professionals, in contrast, although largely highbrow in their tastes, are also able to navigate - to pass as culturally competent within - the world of popular music culture «(Emmison 2003, S. 225-226). Emmison behauptet also, dass die Angehörigen hochqualifizierter Klassenlagen weiterhin einen dominant hochkulturellen Musikgeschmack haben, sich aber in weiten Bereichen der Kultur als kompetent erweisen und diese Kompetenz in unterschiedlichen sozialen Kontexten und Netzwerken als eine Art von >kulturellem Schmiermittel< von Interaktionen und Konversationen nutzen können. Damit schlägt Emmison mit seinem Konzept der kulturellen Mobilität eine deutlich andere theoretische Lesart und vor allem empirische Operationalisierung des Allesfresserphänomens vor, als dies bei Peterson und Kern zu finden ist.

\subsection{Bethany Brysons Studie über musikalische Exklusivität}

Im Anschluss an die Bemerkung von Peterson und Kern, dass kulturelle Allesfresserei nicht bedeute, dass man alle musikalischen Genres gleichermaßen mag, sondern dass dies eine Offenheit für verschiedene Genres anzeige, lässt sich noch eine weitere Lesart des Konzepts der Allesfresserei entwickeln, die hier im Anschluss an die Arbeiten von Bethany Bryson skizziert werden soll. Diese fokussiert in ihren Studien stärker als Emmison auf die tatsächliche Toleranz und Offenheit gegenüber unterschiedlichen Genres (Bryson 1996). Sie fragt also nicht primär danach, wie gut sich die Individuen mit unterschiedlichen kulturellen Genres auskennen oder wie viele Genres sie mögen, sondern sie betrachtet die Anzahl von Genres, gegenüber denen sich die Personen als intolerant erweisen, die sie also ablehnen. Dabei stützt sie sich vor allem auf Studien zum Zusammenhang von Bildung und Toleranz aus dem Bereich der politischen Soziologie. Diese zeigen durchweg, dass höhere gebildete Personen auch eine größere Toleranz an den Tag legen. Anders als im klassischen Modell der Distinktion sollten also hochgebildete Personen keineswegs ausschließlich an Hochkultur orientiert sein und alle anderen Genres verabscheuen, sondern sich als besonders tolerant gegenüber einer großen Zahl von Genres erweisen - freilich ohne diese nun auch gleich besonders zu schätzen (Bryson 1996, S. 886-887). Sie schließt hier in starkem Maße an das von Jackman und Muha (1984) entwickelte Konzept der strukturierten Toleranz an. Dieses behauptet, dass hoch gebildete Personen in erster Linie eine oberflächliche Orientierung an liberaldemokratischen Werten wie Toleranz haben, dagegen aber einen echten sozialen Wandel, der zum Abbau von Ungleichheit führen könnte, ablehnen. Daher schlussfolgert Bryson auch für die musikalische Toleranz, dass diese die Genres ausschließt, die von den Angehörigen unterer Status- oder Bildungsgruppen favorisiert werden (Bryson 1996, S. 887-888). In einer statistischen Analyse des General Social Survey von 1993 kann sie deutliche empirische Unterstützung für ihre Thesen finden: Zum einen lehnen hochgebildete Personen deutlich weniger Genres ab als weniger gebildete Personen, zum anderen wird aber deutlich, dass das Ausmaß der Toleranz von der Sozialstruktur der Hörer eines Genres abhängt: Relativ tolerante Personen sind vor allem gegenüber Genres intolerant, die von Personen mit einer niedrigen Bildung favorisiert werden. In ihrem Sample sind dies vor allem Musikarten wie Gospel, Country und Heavy Metal (Bryson 1996, S. 892). Mit Brysons Studie ist eine dritte Lesart der These vom kulturellen Allesfresser vorgestellt worden, die empirisch relativ erfolgreich ist und in stärkerem Maße als die Analysen von Peterson und Kern nicht auf die Präferenz für zahlreiche musikalische Genres fokussiert, sondern vor allem auf die Toleranz für musikalische Genres.

\subsection{Kulturelle Allesfresser in Deutschland: Neuhoffs Analyse und ihre Probleme}

Die Rezeption der Allesfresser-These im deutschsprachigen Raum hat bisher vorwiegend auf der Ebene der Theoriediskussion stattgefunden (Hartmann 1999; Gebesmair 1998; 2001). Eine systematische Überprüfung der Übertragbarkeit dieses Konzepts auf die Situation in 
Deutschland wurde von Neuhoff (2001) vorgenommen. Seine Studie basiert auf einer Befragung von über 6000 Besuchern von Konzerten unterschiedlicher Art, die 1998 - 1999 in Berlin stattgefunden hat. Dabei orientiert er sich vor allem an der empirischen Vorgehensweise der Untersuchung von Peterson und Kern, berücksichtigt aber auch die Anzahl von abgelehnten populären Musikrichtungen, also einen Indikator für kulturelle Toleranz, den man vor allem in der Studie von Bryson findet. Er vergleicht in seiner Studie hochkulturorientierte und andere Personen im Hinblick auf die Anzahl der von ihnen bevorzugten und im Hinblick auf die von ihnen abgelehnten populären Musikarten. ${ }^{3}$ Dabei kommt er zu dem durchgängigen Ergebnis, dass in Deutschland hochkulturorientierte Personen deutlich weniger populäre Musikgenres mögen als andere Personen und dass auch die Anzahl der Abneigungen gegen populäre Genres in dieser Gruppe höher ist als in anderen Gruppen (Neuhoff 2001, S. 758-762). Neuhoff kommt also zu dem Resultat, dass man die Allesfresser-These von Peterson und Kern nicht auf Deutschland übertragen könne, seine Ergebnisse deuten eher das Gegenteil der These vom kulturellen Allesfresser an, nämlich ein deutliches Überleben der Hochkultursnobs in der Bundesrepublik. Dabei nennt er als Ursache vor allem zwei Faktoren: einerseits die Bedeutung der Countrymusik als schichtübergreifender, aus unterschiedlichen kulturellen Wurzeln stammender, integrativer Musik der Weißen in den Vereinigten Staaten (Neuhoff 2001, S. 768-769), andererseits die Schwäche hochkultureller Institutionen in den Vereinigten Staaten, die nie in gleichem Maße auf öffentliche finanzielle Unterstützung zurückgreifen konnten und daher auch eine viel geringere Dichte entwickeln konnten als in Deutschland (Neuhoff 2001, S. 769-770).

Die Richtigkeit von Neuhoffs Schlussfolgerungen bleibt an dieser Stelle dahingestellt und wird im abschließenden Abschnitt dieses Aufsatzes noch einmal aufgegriffen. Hier soll vor allem darauf verwiesen werden, dass seine Studie auf einer stark verzerrten Stichprobe beruht, die daher auch nicht zur Überprüfung der Übertragbarkeit des Konzepts der kulturellen Allesfresser auf die Bundesrepublik genutzt werden kann. Neuhoff selbst diskutiert die Frage, ob eine Besucherumfrage in Konzerten als empirische Grundlage für eine solche Analyse genutzt werden könne, da damit die Personen in der Bevölkerung, die keine Konzerte besuchen, ausgeschlossen seien (Neuhoff 2001, S. 756, 767-768), verwirft aber mögliche Zweifel mit dem Argument, dass der Vergleich zwischen Subgruppen (Hochkulturorientierte Personen versus Andere) auch in einer verzerrten Stichprobe zu einem mit einer Bevölkerungsumfrage vergleichbaren Resultat führen müsste, freilich auf einem anderen Werteniveau (Neuhoff 2001). Gegen diese Behauptung kann nun allerdings argumentiert werden, dass die Verzerrung von Neuhoffs Daten nicht die gesamte Stichprobe in gleichem Maße betrifft, also einfach eine Verschiebung von Mittelwerten gegenüber der Gesamtbevölkerung bedeutet, sondern für die Subgruppen separat stattfindet. Die hochkulturorientierten Personen in einer Besucherumfrage unterscheiden sich nämlich systematisch von den hochkulturorientierten Personen in der Bevölkerung. In einer Analyse von Konzertbesuchern sind erstens die hochkulturorientierten Personen ausgeschlossen, die aus verschiedenen Gründen keine Konzerte besuchen, vor allem sind zweitens in der Stichprobe aber die häufigen Besucher von Konzerten deutlich überrepräsentiert, was eine einfache Konsequenz aus der Methode der Besucherbefragung ist. Dies bedeutet, dass die Subgruppe der Hochkulturorientierten in Neuhoffs Studie überproportional durch Personen dominiert ist, die häufig in klassische Konzerte gehen. Nun ist aber genau diese Personengruppe - wie auch Neuhoff selbst in seiner Studie feststellt (Neuhoff 2001, S. 764) - besonders intolerant gegenüber populären Musikrichtungen. Insofern muss man wohl schlussfolgern, dass die Daten der Besucherumfrage nicht für eine systematische Überprüfung der Allesfresser-These für Deutschland geeignet sind.

3) Dabei geht er ausgesprochen differenziert vor und entwickelt verschiedene Operationalisierungen für Hochkulturorientierung und für kulturelle Allesfresserei. 
Die Problematik der Studie kann auch durch Daten des ALLBUS von 1998 belegt werden, die also fast zeitgleich mit Neuhoffs Besucherumfrage erhoben worden sind. Im ALLBUS wurden die Vorlieben für lediglich fünf Musikrichtungen abgefragt, so dass die Spannbreite musikalischer Vorlieben nur eingeschränkt analysiert werden kann. Um die Übertragbarkeit der Allesfresser-These von Peterson und Kern auf die deutsche Situation mit diesen Daten zu überprüfen, habe ich die Befragten auf der Grundlage ihrer Vorliebe für klassische Musik in zwei Gruppen eingeteilt. Die Befragten, die gerne klassische Musik hören, werden hier als eher hochkulturorientierte Personen betrachtet. Nun kann im zweiten Schritt betrachtet werden, wie viele der populären Musikrichtungen (Volksmusik, Schlager, Pop/Rock, Jazz) die beiden betrachteten Gruppen im ALLBUS mögen. Hier stellte sich heraus, dass in Ostdeutschland die hochkulturorientierten Personen durchschnittlich 1,80 populäre Musikgenres schätzen, während die anderen Befragten 1,74 Genres bevorzugen. In Westdeutschland lag das Verhältnis bei 1,69 Genres für die hochkulturorientierten Befragten zu 1,56 Musikarten für die anderen Befragten. ${ }^{4}$ Die Daten aus dem ALLBUS können also Neuhoffs deutliche Ablehnung der Übertragbarkeit der Allesfresser-These auf Deutschland nicht unterstützen; sowohl in Ost- wie in Westdeutschland haben die hochkulturorientierten Personen einen breiteren Musikgeschmack als die anderen Befragten. Freilich ist dieser Unterschied sehr gering, so dass die Ergebnisse zwar nicht Neuhoffs deutliche Falsifizierung der AllesfresserThese unterstützen, aber auch nicht gerade für eine deutlich größere Spannbreite des Geschmacks bei den hochkulturorientierten Personen in Deutschland sprechen. Das Resultat zieht aber die empirische Grundlage von Neuhoffs Schlussfolgerung in Zweifel. Dies wird auch deutlich, wenn man den Anteil von Hochkultursnobs - also Personen, die nur hochkulturelle Musikrichtungen und keine anderen schätzen - in Neuhoffs Analyse und im ALLBUS miteinander vergleicht: selbst in der offensten Operationalisierung finden sich unter Neuhoffs Besuchern immerhin 22,9\% Hochkultursnobs (Neuhoff 2001, S. 759), dagegen machen diese im ALLBUS lediglich 4,1 \% der Befragten aus. Insofern zeigt sich deutlich, dass mit den Daten der Besucherumfrage keine Überprüfung der Übertragbarkeit des Allesfresserkonzepts auf Deutschland möglich ist. Eine einfache Analyse unter Verwendung von Daten aus dem ALLBUS von 1998 kann Neuhoffs deutliche Ablehnung jedenfalls nicht unterstützen.

\subsection{Konsequenzen für eine empirische Analyse von Kinobesuchern}

Es hat sich gezeigt, dass das Konzept der kulturellen Allesfresser in Deutschland zwar recht breit rezipiert wurde, dass es aber bisher keine wirklich zuverlässige Einschätzung hinsichtlich der Übertragbarkeit dieser These auf deutsche Verhältnisse gibt. Die Studie von Neuhoff (2001) ist zwar ausgesprochen systematisch, kommt aber aufgrund der verzerrten Datenbasis zu problematischen Resultaten. Eine einfache Analyse auf der Grundlage des ALLBUS von 1998 kann Neuhoffs eindeutige Zurückweisung der Allesfresser-These nicht unterstützen, ergibt aber auch keine deutliche Unterstützung für diese These. In der folgenden empirischen Studie soll daher dieses Konzept in allen vorgestellten Varianten für die Situation in Deutschland untersucht werden. Im Anschluss an die Studie von Emmison berücksichtigt die folgende Analyse auch die Kenntnis von populärkulturellen Genres und Werken, bezugnehmend auf Bryson die Ablehnung dieser künstlerischen Richtungen und im Anschluss an Peterson und Kern die Vorliebe für solche Genres. Im Gegensatz zum großen Teil der bisherigen Literatur über das Allesfresserphänomen wird hier allerdings nicht die Spannbreite des Musikgeschmacks analysiert, sondern die Vorliebe für verschiedene Filmgenres. Damit können zwei Ziele erreicht werden: erstens handelt es sich beim Film um eine Art des künstlerischen Ausdrucks, die einerseits schwächer hierarchisiert ist als die Musik und sich anderer-

4) Nur für Westdeutschland war diese Differenz statistisch (auf dem $1 \%$ Niveau) signifikant. 
seits besonders für unterschiedliche Rezeptionsformen - je nach kulturellem Kapital des Zuschauers - eignet (Barnett/Allen 2000; Baumann 2001). Im Bereich des Films existieren damit für hochkulturorientierte Personen geringere Schwellen zur Ausbildung von Vorlieben für eher populäre Genres. ${ }^{5}$ Wenn also das Phänomen des kulturellen Allesfressers in Deutschland überhaupt zu beobachten ist, dann sollte dieses im Bereich des Films in besonderem Maße der Fall sein. Wenn aber noch nicht einmal hier deutliche Anzeichen dafür entdeckt werden können, dann kann die Suche nach den kulturellen Allesfressern in Deutschland wohl aufgegeben werden. Zweitens bedeutet eine empirische Analyse des Filmgeschmacks aber auch einfach eine Erweiterung der bisherigen Diskussion, die sich in erstaunlichem Maße auf den Musikgeschmack konzentriert hat.

\section{Daten und Methode}

Die Studie beruht auf Daten aus einer schriftlichen Befragung von 750 Kinobesuchern in Leipzig. Um ein möglichst breites Spektrum von Besuchern zu erfassen, wurde die Erhebung mittels einer schriftlichen Umfrage einerseits in unterschiedlichen Filmen in einem Multiplexkino (610 Befragte), andererseits in einem größeren Programmkino (140 Befragte) durchgeführt. Für jede Veranstaltung wurde eine Vollerhebung angestrebt, die Verweigerungsquote lag bei ca. 5\%. Die Fragebögen wurden zusammen mit einem Teilnahmeanreiz vor dem Film ausgeteilt und nach dem Film wieder eingesammelt. Von 950 verteilten Erhebungsbögen wurden 750 ausgefüllt wieder zurückgegeben, so dass eine Rücklaufquote von $79 \%$ erreicht werden konnte. Der Fragebogen enthielt Fragen zum Filmgeschmack, zum Rezeptionsverhalten, zu anderen lebensstilrelevanten Vorlieben und zu den sozialstrukturellen Merkmalen der Befragten. Daher konnten auf dieser Grundlage alle im Rahmen der Diskussion verwendeten Konstrukte auch empirisch operationalisiert werden.

Die Unterscheidung zwischen hochkulturorientierten Personen und anderen Befragten wurde analog zu der Studie von Peterson und Kern anhand der Vorliebe für klassische Musik durchgeführt. Da es sich bei der Musik um ein stark hierarchisiertes Gefüge von Genres handelt, eignet sie sich in besonderem Maße für die Klassifikation von Personen (Bourdieu 1982, S. 41; Gebesmair 2001). Zudem können damit vergleichbare Probleme wie in Neuhoffs Studie vermieden werden. Einerseits sind natürlich auch in dieser Besucherumfrage die Nichtbesucher unberücksichtigt und die häufigen Besucher überrepräsentiert, aber andererseits gibt es keine subgruppenspezifische Verzerrung, da die Definition der Subgruppen durch die musikalischen Präferenzen und nicht durch filmspezifische Indikatoren vorgenommen wurde. Die verschiedenen Konstrukte für das Konzept des kulturellen Allesfressers wurden aber anhand von Indikatoren für den Bereich des Films operationalisiert (vgl. Beer 2000; Neckermann 1997; Berg/Frank 1979; Henseler 1987). Dazu konnte auf zwei Itembatterien im Fragebogen zurückgegriffen werden: Erstens gab es eine Liste von zwanzig Genres (vgl. Abbildung 1); die Befragten konnten im Hinblick auf diese Filmarten zwei Informationen geben: einerseits ob ihnen diese bekannt sind und andererseits ihre Präferenz auf einer vierstufigen Skala. Zweitens wurde eine Liste von zwölf Filmen zusammengestellt, die in den vergangenen fünf Jahren in den Kinos liefen. Es wurden relativ bekannte Filme mit höheren Besucherzahlen ausgewählt, damit diese den Befragten nicht völlig unbekannt sind und zudem wurde ein möglichst breites Spektrum von Filmarten operationalisiert. ${ }^{6}$ Die Be-

5) Dazu kommt noch die Tatsache, dass in Kinos vor allem jüngere Personen anzutreffen sind, bei denen das Phänomen der kulturellen Allesfresserei eher zu finden ist (Peterson/Kern 1996; Neuhoff 2001).

6) Dabei handelte es sich um folgende Filme: (T)Raumschiff Surprise, The Hours, Apocalypse Now (der zwar im Hinblick auf die Erstaufführung älter ist, aber wenige Jahre vor der Umfrage als Director's Cut in den Kinos lief), 50 erste Dates, Shrek, Bowling for Columbine, Herr der Ringe, Halbe Treppe, Sprich mit Ihr, Smoke, Terminator 3, Das Fest. 
fragten konnten im Hinblick auf diese Filme erstens mitteilen, ob sie sie kennen und zweitens ob sie sie gesehen haben. Die Kombination dieser zwei Typen von Informationen soll häufige Probleme in der Analyse von Phänomenen des kulturellen Geschmacks umgehen: Berücksichtigt man in einer solchen Studie nur verschiedene künstlerische Genres, so weiß der Forscher nicht in jedem Fall, ob die Befragten unter dem Genre das Gleiche verstehen (vgl. Bryson 1996). Andererseits bleiben bei der alleinigen Verwendung von konkreten Beispielen die durch die Bezugnahme auf Filmarten evozierten Verallgemeinerungen und Werturteile bei den Befragten außen vor, so dass eine Kombination der beiden Vorgehensweisen als ratsam erscheint (vgl. insgesamt Gebesmair 2001). Auf dieser Grundlage können nun fünf Operationalisierungen der Konstrukte aus der Diskussion über das Phänomen des kulturellen Allesfressers entwickelt werden: Erstens ist dies die Anzahl der von den Befragten als bekannt angekreuzten Filmgenres, zweitens die Anzahl der als bekannt gekennzeichneten Filmbeispiele; mit diesen beiden Indikatoren kann das Konzept der kulturellen Mobilität von Emmison operationalisiert werden. Als dritter Indikator kann die Anzahl der präferierten Filmgenres berücksichtigt werden, womit die Idee des kulturellen Allesfressers in ihrer von Peterson und Kern vorgestellten klassischen Variante operationalisiert wäre. Ergänzend wurde hier als vierter Indikator die Anzahl der als gesehen angekreuzten Filme aus der Liste der Filmbeispiele berücksichtigt. Fünftens wurde schließlich die Anzahl der Abneigungen gegen Filmgenres als Indikator für die von Bethany Bryson vorgelegte Konzeption des kulturellen Allesfressers gemessen. Damit liegt für den Bereich des Films eine umfassende Operationalisierung der These vom kulturellen Allesfresser vor, die im folgenden Abschnitt einer empirischen Überprüfung unterzogen wird.

\section{Empirische Resultate}

Die Analyse der auf der Grundlage der Kinobesucherumfrage gewonnenen empirischen Daten wird in drei Schritten vorgenommen. Im ersten Schritt werden analog zur Vorgehensweise von Peterson und Kern einerseits, Neuhoff andererseits hochkulturorientierte Befragte und andere Befragte im Hinblick auf die fünf schon erläuterten Indikatoren miteinander verglichen. Damit kann die Übertragbarkeit des Konzepts der kulturellen Allesfresser am Beispiel des Filmgeschmacks untersucht werden. Zweitens soll aber auch analysiert werden, welche Position in der Sozialstruktur Personen mit einem besonders breiten kulturellen Geschmack einnehmen. Im Anschluss an die Studien von Emmison und Bryson wird hier vor allem auf die Rolle der Bildung fokussiert. Dazu werden multivariate Regressionen berechnet, bei der die fünf Indikatoren die abhängige Variable darstellen. Schließlich soll drittens auch Brysons These überprüft werden, dass kulturelle Toleranz diejenigen Genres ausschließt, die von besonders status- oder bildungsniedrigen Bevölkerungsgruppen geschätzt werden.

Welche Resultate erbringt nun die Untersuchung des Filmgeschmacks von Kinobesuchern im Hinblick auf die Frage nach der Übertragbarkeit des Konzepts des kulturellen Allesfressers auf die Verhältnisse in der Bundesrepublik? ${ }^{7}$ Die Ergebnisse dazu sind in Tabelle $1 \mathrm{ab}-$ gebildet. Der bivariate Vergleich zwischen hochkulturorientierten Personen und anderen Befragten zeigt für alle fünf Indikatoren in die von diesem theoretischen Konzept vorhergesagte Richtung: hochkulturorientierte Personen kennen mehr Filmarten und Beispielfilme, sie haben mehr Beispielfilme gesehen, sie haben eine breitere Vorliebe für Filmgenres und sind gegenüber einer größeren Anzahl von Filmarten tolerant. Allerdings sind die substantiellen

7) Die Besucherumfrage wurde zwar in Form einer Lokalstudie in Leipzig durchgeführt, doch dürfte dies ihre Generalisierbarkeit im Hinblick auf die Ausgangsfrage nur bedingt einschränken. Einerseits wurde am Beispiel der Musikpräferenzen deutlich, dass hier die Unterschiede zwischen Ost- und Westdeutschland eher geringfügig sind, zweitens stellt sich die Kinosituation in Leipzig nicht als besonders exzeptionell dar (Seidel 2004). 
Unterschiede zwischen den beiden Vergleichsgruppen doch sehr gering. Die deutlichsten Differenzen finden sich bei der Anzahl der präferierten Filmgenre und bei der Anzahl der tolerierten Filmgenres. Insofern zeigt sich auch in der vorliegenden Studie nur eine recht schwache Evidenz für die Übertragbarkeit des Allesfresserkonzepts auf deutsche Verhältnisse. Es bleibt damit wohl festzuhalten, dass hochkulturorientierte Personen in Deutschland zwar überwiegend keine Hochkultursnobs mehr sind, sie sich andererseits aber auch nicht durch eine besonders hohe Ausprägung der kulturellen Allesfresserei auszeichnen.

Tabelle 1: Indikatoren der kulturellen Vielfalt im Vergleich

\begin{tabular}{llllll}
\hline & $\begin{array}{l}\text { Filmgenre } \\
\text { Kenntnis }\end{array}$ & $\begin{array}{l}\text { Filmgenre } \\
\text { Vorlieben }\end{array}$ & $\begin{array}{l}\text { Filme } \\
\text { Kenntnis }\end{array}$ & $\begin{array}{l}\text { Filme Be- } \\
\text { such }\end{array}$ & $\begin{array}{l}\text { Filmgenre } \\
\text { Toleranz }\end{array}$ \\
\hline Hochkulturorientierte & 19,40 & 8,21 & 8,75 & 4,70 & 15,88 \\
Andere & 19,16 & 7,25 & 8,24 & 4,36 & 15,12 \\
Differenz & 0,24 & 0,96 & 0,51 & 0,34 & 0,76 \\
$\mathrm{~N}$ & 600 & 410 & 627 & 627 & 410 \\
Signifikanzniveau & $\mathrm{p}<0,1$ & $\mathrm{p}<0,01$ & $\mathrm{p}<0,05$ & $\mathrm{p}<0,05$ & $\mathrm{p}<0,05$ \\
Anzahl Genre/Filme & 20 & 20 & 12 & 12 & 20 \\
\hline
\end{tabular}

Damit kommen wir zum zweiten Schritt der statistischen Analyse, der Untersuchung von Zusammenhängen zwischen einerseits den Indikatoren für das Phänomen des kulturellen Allesfressers und andererseits den sozialstrukturellen Positionen der Befragten. Dabei wurde die Bildung der Befragten als zentraler Indikator für ihr kulturelles Kapital berücksichtigt, darüber hinaus aber auch, um die Sozialisation im Herkunftshaushalt zu berücksichtigen, die Bildung der Eltern. Da in zahlreichen Lebensstilstudien neben der Bildung sich vor allem das Alter und das Geschlecht als zentrale Determinanten erwiesen haben (vgl. Otte 2004; Schulze 1992; Müller-Schneider 2000) wurden diese hier auch als erklärende Variablen berücksichtigt. Darüber hinaus wurde auch die Gruppeneinteilung der Befragten in hochkulturorientierte Personen und Andere beibehalten. Die in Tabelle 2 dargestellten Ergebnisse sind rasch zusammengefasst: Erstens zeigt sich auch unter Kontrolle der sozialstrukturellen Position der Befragten der in Tabelle 1 festgestellte Unterschied zwischen hochkulturorientierten und anderen Personen; dieser ist in drei von fünf Fällen statistisch signifikant. Zweitens ergeben sich aber nur ganz wenige Hinweise auf statistische Zusammenhänge zwischen den fünf Indikatoren einerseits und der sozialstrukturellen Position der befragten Personen andererseits. Dieses Resultat stellt nun allerdings die These von Peterson und Kern, dass ein vielfältiger Geschmack und nicht mehr der Hochkultursnobismus in gegenwärtigen Gesellschaften Grundlage für Prozesse der sozialen Distinktion sei, stark in Frage. Zumindest ergeben die in Tabelle 2 dargestellten Analysen keinerlei Hinweis auf eine soziale Grundlage für Prozesse der Distinktion durch einen breiten Geschmack im Sinne des Phänomens der kulturellen Allesfresser. 
Tabelle 2: Multivariate Analyse: Sozialstruktur und kulturelle Vielfalt

\begin{tabular}{llllll}
\hline & $\begin{array}{l}\text { Filmgenre } \\
\text { Kenntnis }\end{array}$ & $\begin{array}{l}\text { Filmgenre } \\
\text { Vorlieben }\end{array}$ & $\begin{array}{l}\text { Filme } \\
\text { Kenntnis }\end{array}$ & $\begin{array}{l}\text { Filme Be- } \\
\text { such }\end{array}$ & $\begin{array}{l}\text { Filmgenre } \\
\text { Toleranz }\end{array}$ \\
\hline Konstante & $19,25^{* * *}$ & $9,18^{* * *}$ & $8,10^{* * *}$ & $3,33^{* * *}$ & $14,30^{* * *}$ \\
& $(44,20)$ & $(9,30)$ & $(13,10)$ & $(6,53)$ & $(14,09)$ \\
Hochkultur & 0,05 & $0,18^{* * *}$ & $0,08^{*}$ & 0,07 & $0,11^{*}$ \\
& $(1,20)$ & $(3,65)$ & $(1,97)$ & $(1,60)$ & $(2,17)$ \\
Bildungsniveau & 0,02 & $-0,14 * *$ & $0,10^{*}$ & $0,09 *$ & $-0,04$ \\
& $(0,41)$ & $(-2,64)$ & $(2,30)$ & $(2,04)$ & $(-0,80)$ \\
Bildungsniveau des Va- & 0,04 & $-0,02$ & 0,04 & 0,06 & $-0,05$ \\
ters & $(0,80)$ & $(-0,40)$ & $(0,89)$ & $(1,21)$ & $(-0,77)$ \\
Bildungsniveau der Mut- & $-0,002$ & 0,03 & 0,04 & $-0,02$ & $0,17 * *$ \\
ter & $(-0,04)$ & $(0,47)$ & $(0,84)$ & $(-0,40)$ & $(2,68)$ \\
Alter & 0,03 & $-0,04$ & $-0,13 * *$ & $-0,06$ & 0,05 \\
& $(0,62)$ & $(-0,71)$ & $(-2,92)$ & $(-1,34)$ & $(0,94)$ \\
Geschlecht & $-0,10^{*}$ & 0,01 & $-0,04$ & $0,09 *$ & 0,00 \\
& $(-2,48)$ & $(0,21)$ & $(-1,13)$ & $(2,18)$ & $(0,002)$ \\
Korrigiertes $\mathrm{R}^{2}$ & 0,008 & 0,032 & 0,036 & 0,016 & 0,019 \\
$\mathrm{~N}$ & 600 & 410 & 627 & 627 & 410 \\
\hline
\end{tabular}

Angegeben sind die standardisierten Regressionskoeffizienten und die t-Werte (in Klammern).

$*=\mathrm{p}<0,05 ; * *=\mathrm{p}<0,01 ; * * *=\mathrm{p}<0,001$

Damit kann nun abschließend im Anschluss an die Diskussion von Bryson (1996) betrachtet werden, ob die Toleranz für Filmgenres diejenigen Genres ausschließt, die vor allem von status- und bildungsniedrigen Personen bevorzugt werden. Basierend auf der hier weitgehend replizierten Vorgehensweise von Bryson wurde daher für jedes Filmgenre eine logistische Regression berechnet: als erklärende Variablen wurde die Anzahl der tolerierten Filmarten (ausschließlich des als abhängige Variable betrachteten Genres) und die Bildung der Befragten verwendet. Die geschätzten Parameter für diese Variablen wurden für alle Genres in der folgenden Abbildung 1 abgetragen. Dabei sind die Filmarten nach dem Bildungseffekt sortiert: Personen mit hoher Bildung lehnen also selten Literaturverfilmungen, Satiren und Autorenfilme ab, dagegen lehnen sie besonders oft Horrorfilme, Thriller und Komödien ab. Die Linie für die Effekte der Anzahl der tolerierten Filmgenres auf die Ablehnung eines jeweils spezifischen Genres zeigt kaum relevante Unterschiede. Personen, die viele Filmarten tolerieren, machen ganz offensichtlich keine großen Unterschiede zwischen verschiedenen Typen von Filmen. Damit ist auch für Brysons These von der strukturierten Toleranz, die alle Genres ausschließt, die vor allem von Personen mit niedrigem Status oder niedriger Bildung bevorzugt werden, keine Evidenz zu finden. Dies deckt sich auch mit den Ergebnissen des zweiten Analyseschrittes, der kaum Zusammenhänge zwischen der Ausprägung des Allesfresserphänomens einerseits und der sozialstrukturellen Position der Befragten andererseits erbracht hat. 
Abbildung 1: Der Einfluss von Bildung und Toleranz auf die Ablehnung von unterschiedlichen Filmgenres

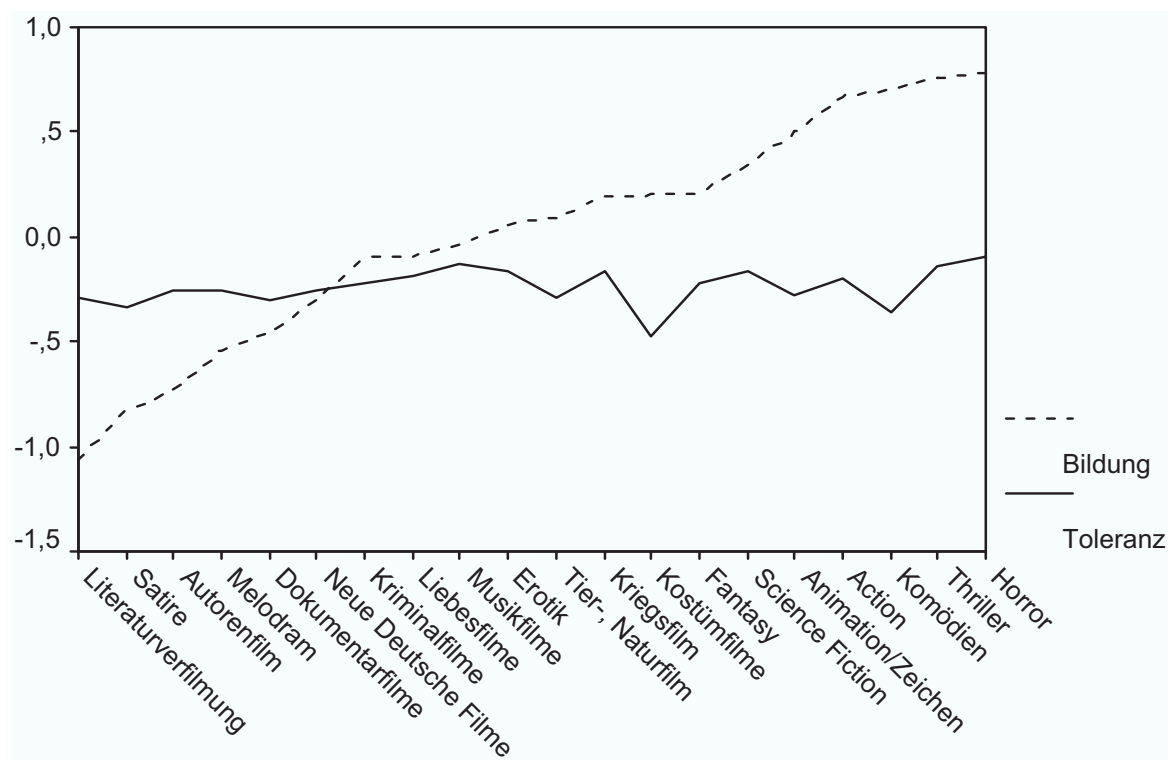

\section{Zusammenfassung und Diskussion}

Als eine wichtige These über den Wandel der sozialen Distinktion in gegenwärtigen Gesellschaften hat sich die Idee vom sogenannten kulturellen Allesfresser durchgesetzt (Peterson/ Kern 1996). Dieses Konzept behauptet, dass in gegenwärtigen Gesellschaften Prozesse der Distinktion vor allem auf geschmacklicher Vielfalt beruhen und nicht mehr auf einer snobistischen Hochkulturorientierung, wie sie z. B. noch von Bourdieu vertreten wurde (Bourdieu 1982). Im Lauf der Diskussion über das Konzept wurden allerdings verschiedene Möglichkeiten zur theoretischen Ausgestaltung und empirischen Operationalisierung desselben entwickelt, die hier im Überblick vorgestellt wurden. Erstens der Fokus auf die tatsächliche Anzahl von präferierten populären Genres (Peterson/Kern 1996), zweitens der Fokus auf die Kenntnis von populären Genres (Emmison 2003) und drittens die Toleranz gegenüber einer Vielzahl von künstlerischen Richtungen (Bryson 1996). Ob sich diese, vor allem im USamerikanischen Kontext entwickelten Deutungen auch auf die Verhältnisse in der Bundesrepublik übertragen lassen, ist umstritten. Vor allem Neuhoff (2001) hat auf der Basis von Daten aus einer großangelegten Besucherumfrage die Anwendbarkeit dieses theoretischen Konzepts in Deutschland scharf zurückgewiesen, da er durchgängig zu Resultaten gelangt ist, die der Allesfresser-These entgegengesetzt waren. Für dessen Studie konnte hier allerdings gezeigt werden, dass die Datengrundlage hochgradig verzerrt und daher für den Zweck der Untersuchung unangemessen war.

Um eine angemessene Überprüfung der Übertragbarkeit des Allesfresserkonzepts auf die bundesdeutschen Verhältnisse vorzunehmen, wurde hier eine Analyse von Daten aus einer Befragung von Kinobesuchern vorgenommen. Mit der Auswahl des Films als künstlerischer Sparte wird einerseits ein Feld gewählt, das diesem Konzept außerordentlich entgegenkommt und das andererseits die empirische Diskussion über das Allesfresserkonzept deutlich verbreitert. In dieser Studie wurden alle drei vorgestellten Varianten des Allesfresserkon- 
zepts empirisch operationalisiert. Dabei wurden im Anschluss an die Studie von Peterson und Kern (1996) hochkulturorientierte und andere Personen unter den Befragten im Hinblick auf ihren Grad von kultureller Allesfresserei verglichen. Zwischen den betrachteten Gruppen gab es bei allen verwendeten Indikatoren statistisch signifikante Unterschiede in der von diesem Konzept vorhergesagten Richtung. Berücksichtigt man aber darüber hinaus, dass mit dem Film eine künstlerische Sparte betrachtet wurde, die dieser These besonders entgegenkommt, dann blieben die substantiellen Unterschiede auf einem erstaunlich niedrigen Niveau. Auch wenn daher die Übertragbarkeit der Idee von Peterson und Kern nicht so deutlich zurückgewiesen werden kann, wie von Neuhoff (2001) vermutet, so sprechen die Ergebnisse nicht für einen deutlichen - die Allesfresser-These bestätigenden - Unterschied zwischen hochkulturorientierten Personen und anderen Personengruppen in Deutschland.

Dieses Fazit wird noch unterstützt durch weitere empirische Analysen. Erstens konnten für das hier analysierte Beispiel des Films in Deutschland die Ergebnisse von Emmison und Bryson über die sozialstrukturelle Lagerung des Phänomens der kulturellen Allesfresser nicht repliziert werden. Für keinen der hier verwendeten Indikatoren dieses Konstrukts ergab sich eine deutliche sozialstrukturelle Lagerung. Damit fehlt aber jede soziale Grundlage für die Prozesse der Distinktion, die auf dem Phänomen der kulturellen Allesfresserei aufruhen sollen. Dies gilt auch für eine weitere These, die vor allem von Bethany Bryson verfochten wurde. Diese behauptet, dass die Toleranz gegenüber kulturellen Genres dort aufhört, wo Genres vor allem von status- oder bildungsniedrigen Personen bevorzugt werden. Auch diese Behauptung konnte in der hier vorgenommenen Studie nicht unterstützt werden. Kulturell tolerante Personen machen laut diesen Ergebnissen keine großen Unterschiede zwischen den verschiedenen Filmarten.

Damit ergibt sich als Zusammenfassung der präsentierten Studie eine äußerst skeptische Einschätzung hinsichtlich der Übertragbarkeit des Konzepts der kulturellen Allesfresser in seinen verschiedenen Varianten auf die kulturelle und soziale Situation in Deutschland. Dabei darf freilich nicht übersehen werden, dass die vorliegenden Ergebnisse im Gegensatz zu Neuhoffs Studie auch nicht für eine einfache Fortexistenz des Hochkultursnobismus sprechen, sondern für eine dosierte Grenzüberschreitung zwischen einem hochkulturellen und einem populären Geschmack. Vor allem bei Personengruppen mit einem Alter von über 40 Jahren finden sich z.B. Hinweise darauf, das hier hochkulturelle Neigungen zum Teil mit Elementen des sogenannten Trivialschemas (Schulze 1992) angereichert werden, deutlich erkennbar im Genre der Operette. Diese Form der Grenzüberschreitung tritt bei den jüngeren Altersgruppen allerdings eher selten auf, hier kommt es dagegen häufiger zu einer Vermischung von Hochkulturschema und Spannungsschema (Gebesmair 2004). Wichtig für die zukünftige Forschung ist also vor allem eine differenzierte Analyse der spezifischen symbolischen Überschreitungen der ehemals so stabilen Grenze zwischen Hoch- und Populärkultur, ihren sozialen Grundlagen und spezifischen Ausformungen (Müller-Schneider 1996; 2000; Schulze 1992; Gebesmair 2004; Otte 2004; Rössel/Beckert-Zieglschmid 2002).

Ansätze für eine mögliche und plausible Erklärung für die Nichtübertragbarkeit der Allesfresser-These auf Deutschland bietet Neuhoff in seinem Aufsatz. Erstens verweist er auf das Phänomen der Countrymusik, die in den Vereinigten Staaten quer durch alle soziale Gruppen geschätzt wird und stärker als die klassische Musik eine Art von legitimer Kultur des weißen Amerika bildet (Neuhoff 2001). Vor allem die Studie von Bryson führt zu Skepsis gegenüber diesem Argument, da nun gerade die Countrymusik zu den am stärksten abgelehnten Genres gehört (Bryson 1996: 892). In einer verallgemeinerten Form könnte dieses Argument aber durchaus plausibel sein, wenn man berücksichtigt, dass in den Vereinigten Staaten die Akzeptanz von populärer Musik und Kultur allgemein sehr viel größer ist als in Europa. Daher ist das Phänomen des Hochkultursnobismus dort auch kulturell ausgesprochen randständig. In ihren Daten können Peterson und Kern daher 1982 nur 10 und 1992 nur 
3 Hochkultursnobs finden (Person/Kern 1996, S.901) und dies unter insgesamt über 11000 Befragten! Auch schon in den frühen sechziger Jahren konnte Wilensky in einer Studie in Detroit und Umgebung keine Person finden, die ein perfekter Hochkultursnob gewesen wäre (Wilensky 1964). Damit spricht sehr viel für die These, dass unabhängig von Prozessen des sozialen und kulturellen Wandels in der amerikanischen Gesellschaft die Akzeptanz von populärer Kultur sehr viel breiter ist und daher Hochkultursnobismus selbst in den sozialen Eliten nie ein besonders weit verbreitetes Phänomen war. Insofern scheint hier ein dauerhafter Unterschied zu den kulturellen Präferenzen in Deutschland zu existieren. Dies kann wiederum mit dem zweiten von Neuhoff formulierten Argument zusammenhängen, dass nämlich die hochkulturelle Angebotsstruktur in den Vereinigten Staaten sehr viel spärlicher ausgeprägt ist als in Deutschland, wo hunderte von Musikschulen, Orchestern und Konzerthäusern für eine weltweit nahezu einzigartige, flächendeckende Versorgung mit klassischer Musik sorgen. Ganz im Sinne der kulturellen Produktionsfelder von Pierre Bourdieu (1999) ist die Hochkultur in der Bundesrepublik Deutschland flächendeckend institutionalisiert: nicht nur die Orte des aktiven und passiven Konsums von klassischer Musik gehören dazu, auch die Dominanz anspruchsvoller Literatur und Musik im Schulunterricht, der hochkulturelle Fokus im Feuilleton und nicht zuletzt die unhinterfragte Vorrangstellung der Förderung von Hochkultur im Bereich der Kulturpolitik unterstützten die Stabilisierung und Reproduktion hochkultureller Geschmacksvorlieben in Teilen der Bevölkerung. Mit diesem Argument ist auch eine wichtige Ausweitung der Forschung über soziale Distinktion, Lebensstile und Sozialstruktur verbunden: Die Forschung darf nicht länger allein auf die Sozialstruktur und die Lebensstile der Nachfrager und Konsumenten von Kultur fokussieren, sondern sie muss stärker auch die Struktur und Dynamik der Anbieter von Kultur in ihren Modellen berücksichtigen. Die Entwicklung von Lebensstilen und Geschmackspräferenzen ist nicht allein ein Produkt der Veränderung der Sozialstruktur und der individuellen Werte, sondern kann auch ein Resultat der Institutionalisierung bestimmter Formen der Kulturproduktion und der jeweiligen Struktur der Märkte für bestimmte kulturelle Angebote sein (Peterson/Berger 1975; DiMaggio 1982; 1987a; 1987b). Gerade im internationalen Vergleich von Lebensstilen und kulturellen Vorlieben dürfte die jeweilige regionale oder nationale Angebotsstruktur von erheblicher Bedeutung sein.

\section{Literatur}

Barnett, Lisa A. / Allen, Michael P. (2000): Social Class, Cultural Repertoires, and Popular Culture: The Case of Film, in: Sociological Forum 15, S. 145-163.

Beer, Carolin (2000): Die Kinogeher. Eine Untersuchung des Kinopublikums in Deutschland, Berlin: Vistas.

Bennett, Tony / Emmison, Michael / Frow, John (1999): Accounting for Tastes: Australian Everyday Cultures, Cambridge: Cambridge UP.

Berg, Elisabeth / Bernward, Frank, (1979): Film und Fernsehen. Ergebnisse einer Repräsentativerhebung 1978, Mainz: Haase \& Köhler.

Bourdieu, Pierre (1982): Die feinen Unterschiede. Kritik der gesellschaftlichen Urteilskraft, Frankfurt/ Main: Suhrkamp.

Bourdieu, Pierre (1999): Die Regeln der Kunst. Genese und Struktur des literarischen Feldes, Frankfurt/ Main: Suhrkamp.

Bryson, Bethany (1996): Anything but Heavy Metal. Symbolic Exclusion and Musical Dislikes, in: American Sociological Review 61, S. 884-899.

DiMaggio, Paul (1982): Cultural Entrepreneurship in Nineteenth Century Boston, Part I: The Creation of an Organizational Base for High Culture in America, in: Media, Culture and Society 4, S. 33-50.

DiMaggio, Paul (1987a): Classification in Art, in: American Sociological Review 52, S. 440-455. 
DiMaggio, Paul (1987b): Nonprofit Organizations in the Production and Distribution of Culture, in: Walter W. Powell (Hrsg.): The Nonprofit Sector. A Research Handbook, New Haven: Yale University Press, S. 195-200.

Emmison, Michael (2003): Social Class and Cultural Mobility: Reconfiguring the Cultural Omnivore Thesis, in: Journal of Sociology 39, S. 211-230.

Gebesmair, Andreas (1998): Musikgeschmack und Sozialstruktur: Zum Begriff Omnivore in der amerikanischen Kultursoziologie der 90er Jahre, in: Österreichische Zeitschrift für Soziologie 23, S. 5-22.

Gebesmair, Andreas (2001): Grundzüge einer Soziologie des Musikgeschmacks, Wiesbaden: Westdeutscher Verlag.

Gebesmair, Andreas (2004): Renditen der Grenzüberschreitung. Zur Relevanz der Bourdieuschen Sozialtheorie für die Analyse sozialer Ungleichheiten, in: Soziale Welt 5, S. 181-204.

Hartmann, Peter H. (1999): Lebensstilforschung: Darstellung, Kritik und Weiterentwicklung, Opladen: Leske + Budrich.

Henseler, Stephanie (1987): Soziologie des Kinopublikums. Eine sozialempirische Studie unter besonderer Berücksichtigung der Stadt Köln, Frankfurt/Main: Lang.

Jackman, Mary R. / Muha, Michael J. (1984): Education and Intergroup Attitudes: Moral Enlightenment, Superficial Democratic Commitment, or Ideological Refinement? In: American Sociological Review 49, S. 751-769.

Müller-Schneider, Thomas (1996): Wandel der Milieulandschaft in Deutschland. Von hierachisierenden zu subjektorientierten Wahrnehmungsmustern, in: Zeitschrift für Soziologie 25, S. 190-206.

Müller-Schneider, Thomas (2000): Stabilität subjektorientierter Strukturen. Das Lebensstilmodell von Schulze im Zeitvergleich, in: Zeitschrift für Soziologie 29, S. 361-374.

Neckermann, Gerhard (1997): Der Kinobesuch 1991 bis 1996 nach Besuchergruppen. Fortschreibung des Berichts bis November 1996, Berlin: FFA.

Neuhoff, Hans (2001): Wandlungsprozesse elitärer und populärer Geschmackskultur? Die »AllesfresserHypothese « im Ländervergleich USA/Deutschland, in: Kölner Zeitschrift für Soziologie und Sozialpsychologie 53, S. 751-772.

Otte, Gunnar (2004): Sozialstrukturanalyse mit Lebensstilen, Wiesbaden: Westdeutscher Verlag.

Peterson, Richard A. (1992): Understanding Audience Segmentation: From Elite and Mass to Omnivore and Univore, in: Poetics 21, S. 243-258.

Peterson, Richard A. (1997): The Rise and Fall of Highbrow Snobbery as a Status Marker, in: Poetics 25. S.75-92.

Peterson, Richard A. / Berger, David A. (1975): Cycles in Symbol Production: The Case of Popular Music, in: American Sociological Review 40, S.158-173.

Peterson, Richard A. / Simkus, Albert (1992): How Musical Taste Groups Mark Occupational Status Groups, in: Michele Lamont und Marcel Fournier (Hrsg.): Cultivating Differences, Chicago: University of Chicago Press, 1992, S. 152-168

Peterson, Richard A. / Kern, Roger M. (1996): Changing Highbrow Taste: From Snob to Omnivore, in: American Sociological Review 61, S. 900-907.

Rössel, Jörg / Beckert-Zieglschmid, Claudia (2002): Die Reproduktion kulturellen Kapitals, in: Zeitschrift für Soziologie 31, S. 497-513.

Schulze, Gerhard (1992): Die Erlebnisgesellschaft. Kultursoziologie der Gegenwart, Frankfurt/Main: Campus.

Seidel, Peggy (2004): Blühende Kinolandschaften? Bestandsaufnahme, Konzepte und Analysen der Leipziger Kinoprogramme 2002, Magisterarbeit: Universität Leipzig.

Prof. Dr. Jörg Rössel

Universität Erfurt

Staatswissenschaftliche Fakultät

Lehrstuhl für Allgemeine Soziologie

Nordhäuser Str. 63, D-99089 Erfurt

e-mail: joerg.roessel@uni-erfurt.de 